\title{
Influence des herbicides sur les principaux paramètres physiologiques de la productivité et les composantes essentielles de rendement des blés tendres (Triticum aestivum L.) et durs (Triticum durum Desf.)
}

\author{
Garané Ali ${ }^{*}$, Sawadogo Mahamadou² and Traoré Mamoudou ${ }^{3}$ \\ $1^{*}$ Institut de l'Environnement et de Recherches Agricoles (INERA), CREAF- Kamboinsé, Département Production \\ Végétale, CMFPT, 01 BP 476 Ouagadougou 01, Burkina Faso. \\ 2 UFR/Sciences de la Vie et de la Terre. Université de Ouagadougou, Laboratoire de Génétique et de \\ Biotechnologie Végétales, 03 BP 7021 Ouagadougou 03, Burkina Faso. \\ ${ }^{3}$ Institut de l'Environnement et de Recherches Agricoles (INERA), CREAF-Kamboinsé, Département Gestion \\ des Ressources Naturelles/Système de Production, 01 BP 476 Ouagadougou 01, Burkina Faso. \\ *Auteur correspondant, E-mail: ali_garane@yahoo.fr
}

Original submitted in on 26 th November 2014. Published online at www.m.elewa.org on $28^{\text {th }}$ February 2015 http://dx.doi.org/10.4314/jab.v86i1.9

\section{RESUME}

Objectif: L'objectif de cette étude est d'évaluer les effets de la combinaison du 2,4-D+Lontrel et du Quartz super (diflufénicanil-50g/l+isoproturon-500g/l) sur les principaux paramètres physiologiques de la productivité des blés d'écologies différentes dans la région de Moscou: la surface foliaire, le rendement biologique, l'accumulation journalière de la matière sèche et le rendement grain. Les composantes essentielles de la structure de rendement comme le nombre de plants $/ \mathrm{m}^{2}$, le nombre grains/plant, le poids grains/plant, le poids grains $/ \mathrm{m}^{2}$ et le poids de 1000 grains ont aussi été évalués.

Méthodologie et résultats: Le dispositif expérimental utilisé est un Split plot à quatre répétitions comprenant chacune un témoin (sans herbicide) et trois traitements herbicides: le Quartz super (dose 1,0 I ha-1 m. a.) appliqué deux jours après semis et au tallage des blés, la combinaison 2,4-D (dose 1,8 $\mathrm{kg} \mathrm{ha}^{-1} \mathrm{~m}$. a.) + Lontrel (dose $0,3 \mathrm{~kg} \mathrm{ha}^{-1} \mathrm{~m}$. a.) utilisée seulement au tallage. Les résultats ont montré l'effet positif des herbicides sur l'accroissement des principaux paramètres physiologiques de la productivité des blés. L'amélioration des paramètres physiologiques de la productivité a eu un impact important sur la structure de la récolte. Les variétés Lyba, Scham-2, Bykhys-1 et Homari ont enregistré des hausses de rendement grain respectivement de (12.2\%-15.5\%); (5.6\%-6.8\%); (9.0\%-11.1\%) et $(9.8 \%-11.7 \%)$.

Conclusion et application des résultats: II ressort de cette étude que les blés moins concurrentiels aux adventices Lyba (faible tallage) et Homari (tige courte) ont considérablement amélioré les composantes essentielles de leurs rendements comparé aux blés les plus aptes à concurrencer les adventices (Scham-2 et Bykhys-1). Grace aux herbicides, les paramètres physiologiques responsables de la productivité et les composantes de la structure de rendement du blé ont été améliorés contribuant à l'accroissement de rendement grain.

Mots clés: Herbicides, blés, paramètres physiologiques, rendement 
Effects of herbicides on major physiological productivity parameters and essential yield components of performance of varieties of soft wheat (Triticum aestivum L.) and hard wheat (Triticum durum Desf.)

\section{ABSTRACT}

Objective: The objective of this study was to evaluate the effect of the combination of 2,4-D + Lontrel and Quartz super (diflufenican-50 $\mathrm{g} / \mathrm{l}+$ isoproturon - $500 \mathrm{~g} / \mathrm{l}$ ) on major physiological productivity parameters of wheat of different ecologies in the Moscow region: also to the effect of the combination on leaf area, biological yield, the daily accumulation of dry matter and grain yield of soft and hard wheat. The essential yield components of the structure of wheat: the number of seeds/plant, grain weight/plant, the weight of 1000 grains and grain weight $/ \mathrm{m}^{2}$ were evaluated.

Methodology and results: The experimental design used was a split plot design with four replications each comprising a control (no herbicide) and three herbicides: the Quartz super dose (1.0 $\left.\mathrm{lha}^{-1}\right)$ applied two days after sowing and tillering of wheat, the combination of 2,4-D (1.8 kg ha-1)+Lontrel $\left(0.3 \mathrm{~kg} \mathrm{ha}^{-1}\right)$ used only tillering. From the results, it appears that the use of herbicides increase major physiological productivity parameters of wheat. Improvement of physiological productivity parameters by herbicides applied had a significant impact on the structure of the harvest. The varieties Lyba, Scham-2, Bykhys-1 and Homari have increased in grain yield respectively of $12.2 \%$ to $15.5 \% ; 5.6 \%$ to $6.8 \% ; 9.0 \%$ to $11.1 \%$ ) and $9.8 \%$ to $11.7 \%$.

Conclusion and application of results: Thus, it appears from this study that the wheat varieties less competitive with weeds Lyba (low tillering) and Homari (short stem) greatly improved the essential components of their performance compared to the more competitive (Scham -2 and Bykhys-1) wheat. By the use of herbicides, physiological parameters of plant productivity and yield components of the structure of wheat were enhanced leading to increase of grain yield.

Key words: Herbicides, wheat, physiological parameters, yield

\section{INTRODUCTION}

Le potentiel de productivité des cultures pourrait être apprécié à travers leur capacité photosynthétique (Nichiporovich, 1961; Chatilov et al., 1987). Aussi, le développement et la formation de la surface foliaire des blés dépendent du potentiel photosynthétique. Ce paramètre essentiel est en rapport avec les rendements biologique et économique du blé (Chatilov et al., 1987). Cependant, la masse biologique formée ne reflète pas toujours le rendement de la culture car très souvent les conditions de culture apportent leurs correctives par la réduction ou l'augmentation de la productivité photosynthétique. Les adventices sont souvent cités parmi les problèmes majeurs dans la culture du blé (Bàrberi, 2002; Tuner et al., 2006). Ils entrent en concurrence avec la culture pour la lumière, les éléments minéraux et la ressource hydrique (Prieur, 2010). Cette concurrence entraine des baisses de productivité, des pertes de rendement et de la qualité de la production (Doré et al., 2007;
Valantin-Morisson et al., 2008). Les mesures préventives de lutte contre les adventices nécessitent la connaissance de la biologie, de l'écologie des adventices et une maîtrise des pratiques culturales afin de perturber le cycle de vie des adventices et favoriser la compétition de la culture vis-à-vis des adventices (ValantinMorisson et al. 2008). Lorsque les moyens de lutte préventive n'ont pas réussi à maîtriser les infestations d'adventices, l'utilisation d'herbicides chimiques comme moyen de lutte curatif est nécessaire (Kurstjens, 2007). L'application d'un herbicide est un acte technique rapide, simple, relativement peu coûteux et d'une très bonne efficacité avec un large spectre d'action (Kurstjens, 2007; Cummins, 2009). Mieux encore, pour Asseng et al. (2002), Ramdani (2004), Farineau and Morot-Gaudry (2006) et Darui et al. (2011), les herbicides permettraient une meilleure réalisation des paramètres physiologiques responsables du rendement potentiel du blé. Dans tout les cas, selon Assem 

de la productivité et les composantes essentielles de rendement des blés tendres (Triticum aestivum $\mathrm{L}$.) et durs (Triticum durum Desf.)

et al. (2006), le rendement du blé devait s'apprécier par la valeur des différentes composantes de sa structure. Par conséquent, un bon rendement serait tributaire du nombre de grains par unité de surface et du poids moyen de grains (Gooding et al., 2000; Bahlouli et al., 2005). Dans ce contexte, l'étude du processus d'installation de la structure de la récolte du blé en fonction du bloc chimique appliqué et des conditions pédoclimatiques représente un intérêt pratique car elle permet d'adapter les façons

\section{MATERIEL ET METHODE}

Le matériel végétal. : Le matériel végétal étudié est composé de quatre(04) variétés de blé dont:

- $\quad$ deux de blé tendre (Lyba et Scham-2) culturales aux conditions concrètes de culture et augmenter le rendement et la qualité du grain. L'objectif de notre étude était d'évaluer les effets combinés de la variété, des herbicides 2,4D+Lontrel et Quartz super sur les principaux paramètres physiologiques de la productivité et les composantes essentielles du rendement des variétés de blés tendres (Triticum aestivum L.) et durs (Triticum durum Desf.) dans la zone de Moscou.

- $\quad$ et deux de blé dur (Bykhys-1 et Homari).

Les caractéristiques essentielles des variétés sont présentées dans le tableau 1.

Tableau 1: Les caractéristiques essentielles des blés.

\begin{tabular}{|c|c|c|c|c|c|}
\hline Type de blé & Variété & Origine & Rendement & Cycle & Caractéristiques essentielles \\
\hline $\begin{array}{l}\text { Blé tendre } \\
\text { (Triticum. } \\
\text { aestivum L, var. } \\
\text { Eryhtrospermum } \\
\text { Kôrn) }\end{array}$ & $\begin{array}{l}\text { Scham- } \\
2\end{array}$ & Russie & $\begin{array}{l}4,0-5,0 \text { tha-1 }^{-1} \\
\text { et plus }\end{array}$ & $\begin{array}{l}\text { Semi- } \\
\text { tardif }\end{array}$ & $\begin{array}{l}\text { Chaume haut }(70-80 \mathrm{~cm}) \text {; épi non aristé } \\
\text { et glabre de } 7-10 \mathrm{~cm} \text { de longueur; poids } \\
\text { de } 1000 \text { grains: } 35-45 \mathrm{~g} \text {; sensible à la } \\
\text { rouille brune (P.triticina) et jaune } \\
\text { ( } P \text {.striiformis) } \\
\text { Chaume suffisamment haut }(80-90 \mathrm{~cm}) \text {; } \\
\text { épi } \\
\text { aristé et glabre long de } 7-9 \mathrm{~cm} \text {; poids de } \\
1000 \text { grains: } 34,1-42,1 \mathrm{~g} \text {; résistant aux } \\
\text { espèces de rouille brune et jaune. }\end{array}$ \\
\hline $\begin{array}{l}\text { Blé dur } \\
\text { (Triticum. durum } \\
\text { Desf., var. } \\
\text { Africanum Kôrn) }\end{array}$ & $\begin{array}{l}\text { Bykhys- } \\
1\end{array}$ & Yémen & $3,0-4,0$ t ha- $^{-1}$ & Tardif & $\begin{array}{l}\text { Chaume suffisamment haut }(78 \text { à } 92 \mathrm{~cm}) \text {; } \\
\text { épi aristé et pubescent, long de } 4-6 \mathrm{~cm} \text {; } \\
\text { poids de } 1000 \text { grains: } 41,5-43,3 \mathrm{~g} \text {; } \\
\text { résistant aux espèces de rouille brune et } \\
\text { jaune et sensible au charançon du grain } \\
\text { (Sitophilus granarius). } \\
\text { Chaume court }(46-66 \mathrm{~cm}) \text {, épi aristé et } \\
\text { non pubescent long de } 4-5 \mathrm{~cm} \text {; le poids } \\
\text { de } 1000 \text { grains: } 35,1-35,1 \mathrm{~g} \text {; résistant aux } \\
\text { espèces de rouille brune et jaune; sensible } \\
\text { au charançon du grain. }\end{array}$ \\
\hline
\end{tabular}

\section{Méthodologie}

Dispositif expérimental: Les expériences ont été conduites dans la ville d'Odinsk sur des parcelles de I'Institut de Recherches Agronomiques de la Région Centrale située dans la zone des «terres non noires» ou «nichernozièm» de Russie de 1998-2000. Les précédents culturaux ont été le blé d'hiver triticale et le Brassica napis L. Les parcelles ont reçu en automne au labour $45 \mathrm{~kg} \mathrm{ha}^{-1}$ de substance active de phosphore $\left(\mathrm{P}_{2} \mathrm{O}_{5}\right)$ sous la forme de super phosphate simple et $70 \mathrm{~kg} \mathrm{ha}^{-1}$ de calcium $\left(\mathrm{K}_{2} \mathrm{O}\right)$ sous forme de sel de calcium. Les engrais azotés ont été appliqués au labour à $60 \mathrm{~kg} \mathrm{ha}^{-1}$ et au tallage des blés à $30 \mathrm{~kg}$ $\mathrm{ha}^{-1}$ sous la forme de silicate d'ammonium. Les normes d'ensemencements pratiquées ont été de 5 millions de grains ha-1 pour chaque variété. La parcelle élémentaire a mesurée $50 \mathrm{~m}^{2}(12,5 \mathrm{~m} \times 4$ $\mathrm{m})$. La surface entière de la parcelle élémentaire a été traitée à l'herbicide et la dimension de la zone d'observation (la parcelle utile) est de $25 \mathrm{~m}^{2}$ (12,5 m de longueur x $2 \mathrm{~m}$ de large). Avant le semis, les grains ont été traités à l'Ethylmercurchloride 

et durs (Triticum durum Desf.)

(granozan) en raison de $2 \mathrm{~kg}$ de substance par tonne de semence et semés en ligne $(16 \mathrm{~cm} \times 4 \mathrm{~cm})$. Le Quartz super (concentré soluble) est dilué à raison de 1,0 I dans 350 I d'eau et appliqué deux jours après semis et au tallage des blés. La combinaison 2,4-D $\left(1,8 \mathrm{~kg} \mathrm{ha}^{-1}\right)+$ Lontrel $\left(0,3 \mathrm{~kg} \mathrm{ha}^{-1}\right)$ a été utilisée seulement au tallage. Toutes les parcelles ont été traitées au Triadimefon $25 \%$ à la dose de $0,6 \mathrm{~kg} \mathrm{ha}^{-1}$ dans 500 I d'eau pour la lutte contre les maladies cryptogamiques. Le dispositif expérimental utilisé est un Split plot à quatre répétitions avec en parcelles principales les quatre (4) variétés de blé et en parcelles secondaires les quatre traitements appliqués comprenant chacun un témoin (sans herbicide) et trois traitements herbicides (tableau 2).

Tableau 2: Schéma expérimental

\begin{tabular}{|c|c|c|c|c|}
\hline \multirow{2}{*}{ Traitements } & \multicolumn{2}{|c|}{$\begin{array}{c}\text { Blés tendres } \\
\text { (Triticum aestivum L.) }\end{array}$} & \multicolumn{2}{|c|}{$\begin{array}{c}\text { Blés durs } \\
\text { (Triticum durum Desf.) }\end{array}$} \\
\hline & Lyba & Scham-2 & Bykhys-1 & Homari \\
\hline $\begin{array}{l}\text { Témoin (sans } \\
\text { herbicides) }\end{array}$ & sans herbicides & sans herbicides & sans herbicides & sans herbicides \\
\hline $\begin{array}{l}\text { Technologie de base } \\
(2,4-\mathrm{D}+\text { Lontrel })\end{array}$ & $\begin{array}{c}1,8+0,3 \mathrm{~kg} \mathrm{ha}^{-1} \mathrm{~m} . \mathrm{a} . \\
\text { (tallage complet) }\end{array}$ & $\begin{array}{c}1,8+0,3 \mathrm{~kg} \mathrm{ha}^{-1} \mathrm{~m} . \mathrm{a} . \\
\text { (tallage complet) }\end{array}$ & $\begin{array}{c}1,8+0,3 \mathrm{~kg} \mathrm{ha}^{-1} \mathrm{~m} . \mathrm{a} . \\
\text { (tallage complet) }\end{array}$ & $\begin{array}{c}1,8+0,3 \mathrm{~kg} \mathrm{ha}^{-1} \mathrm{~m} . \mathrm{a} . \\
\text { (tallage complet) }\end{array}$ \\
\hline $\begin{array}{l}\text { Quartz super: } \\
\text { Diflufénicanil -50 g/l + } \\
\text { Isoproturon - } 500 \mathrm{~g} / \mathrm{l}\end{array}$ & $\begin{array}{l}1,0 \text { I ha-1m.a. } \\
\text { (en pré-levée) }\end{array}$ & $\begin{array}{l}\text { 1,0 I ha-1m.a. } \\
\text { (en pré-levée) }\end{array}$ & $\begin{array}{l}1,0 \text { I ha-1m.a. } \\
\text { (en pré-levée) }\end{array}$ & $\begin{array}{l}\text { 1,0 I ha-1m.a. } \\
\text { (en pré-levée) }\end{array}$ \\
\hline $\begin{array}{l}\text { Quartz super: } \\
\text { Diflufénicanil-50 g/l } \\
\text { +|soproturon - } 500 \mathrm{~g} / \mathrm{l}\end{array}$ & $\begin{array}{l}\text { 1,0 I ha-1m.a. } \\
\text { (tallage complet) }\end{array}$ & $\begin{array}{l}1,0 \text { I /ha- }{ }^{1} \mathrm{~m} . \mathrm{a} . \\
\text { (tallage complet) }\end{array}$ & $\begin{array}{l}1,0 \text { I ha-1m.a. } \\
\text { (tallage complet) }\end{array}$ & $\begin{array}{l}1,0 \text { I ha-1m.a. } \\
\text { (tallage complet) }\end{array}$ \\
\hline
\end{tabular}

Les paramètres mesurés: Pour chaque traitement, 4 placettes de $0,25 \mathrm{~m}^{2}$ ont été délimitées sur lesquelles les différentes variables ont été évaluées. La hauteur de la plante a été mesurée depuis le sol jusqu'au sommet de l'épi de la tige principale. Les nombres de plants et de talles utiles par mètre carré ont été obtenus par comptage durant les principaux stades phénologiques. Les composantes du rendement (poids grain par plante et au mètre carré, poids de 1000 grains et rendement grain) ont été pesé à la récolte. Les paramètres physiologiques

\section{RESULTATS}

Influence des herbicides sur la dynamique de croissance de la surface foliaire des semis de blés tendres (Triticum aestivum L.) et durs (Triticum durum Desf.) : L'évolution des surfaces foliaires au cours de la période végétative des différentes variétés de blé, présentée dans le tableau 3 , montre qu'elle s'est déroulée en fonction des caractéristiques de chaque variété. Ainsi, la surface foliaire maximale dans les traitements témoins (sans herbicides) chez les blés tendres au stade épiaisonfloraison a été de 29,48 milles $\mathrm{m}^{2} \mathrm{ha}^{-1}$ (Lyba) et de 31,02 milles $\mathrm{m}^{2} \mathrm{ha}^{-1}$ (Scham-2), tandis que celle des blés durs (Bykhys-1, Homari) à la même période a été respectivement de 28,05 milles $\mathrm{m}^{2} \mathrm{ha}^{-1}$ et de 25,64 milles $\mathrm{m}^{2} \mathrm{ha}^{-1}$. Les surfaces foliaires maximales essentiels de la productivité des blés (surface foliaire, potentiel photosynthétique des semis, rendement biologique et l'accumulation journalière de matière sèche et l'indice de récolte) de la plante ont été mesurés au cours de la période végétative des blés.

Analyse des données: Les analyses de variance Hierarchical Column Prime Order ont été effectuées avec Minitab version 13.0. Les moyennes ont été comparées pour apprécier si les différences entre les résultats obtenus sont significatives à la probabilité de $5 \%$.

dans les traitements herbicides, ont été en moyenne pour les variétés Lyba et Scham-2 de 30,42 milles $\mathrm{m}^{2}$ ha-1 et 32,51 milles $\mathrm{m}^{2} \mathrm{ha}^{-1}$ et respectivement de 29,46 milles $\mathrm{m}^{2} \mathrm{ha}^{-1}$ et 26,76 milles $\mathrm{m}^{2}$ ha-1 pour les blés Bykhys-1 et Homari. L'amélioration de l'état phytosanitaire des semis grâce aux herbicides a permis aux premiers stades de croissance des blés l'augmentation de la surface foliaire et de la biomasse, ce qui se traduit par une meilleure interception de la lumière. En moyenne, les hausses de l'appareil foliaire ont été de 3,2\% (Lyba) et de $4,8 \%$ (Scham-2). Les blés durs Bykhys-1 et Homari ont connu des hausses de surface foliaire de $5,1 \%$ et $4,3 \%$ respectivement. 
Tableau 3: Influence des herbicides sur la croissance de la surface foliaire des blés tendres et durs (milliers $\mathrm{m}^{2}$ ha-1).

\begin{tabular}{|c|c|c|c|c|c|c|c|c|}
\hline Traitements & $\begin{array}{l}\text { Levée } \\
\text { complète }\end{array}$ & $\begin{array}{l}\text { Tallage } \\
\text { complet }\end{array}$ & Montaison & Épiaison & Floraison & $\begin{array}{l}\text { Maturité } \\
\text { laiteuse }\end{array}$ & $\begin{array}{l}\text { Maturité } \\
\text { cireuse }\end{array}$ & Récolte \\
\hline \multicolumn{9}{|c|}{ Blé tendre : Lyba } \\
\hline $\begin{array}{l}\text { Témoin: sans } \\
\text { herbicides }\end{array}$ & 6,78 & 15,68 & 23,56 & 29,66 & 29,48 & 26,24 & 7,30 & 1,31 \\
\hline $\begin{array}{l}\text { 2,4-D+Lontrel } \\
\text { en post-levée }\end{array}$ & 7,42 & 17,25 & 24,52 & 30,83 & 30,43 & 27,11 & 7,04 & 1,12 \\
\hline $\begin{array}{l}\text { Quartz super } \\
\text { en pré-levée }\end{array}$ & 7,00 & 16,35 & 23,46 & 29,48 & 30,35 & 27,10 & 6,89 & 1,00 \\
\hline $\begin{array}{l}\text { Quartz super } \\
\text { en post-levée }\end{array}$ & 7,26 & 16,82 & 24,45 & 30,88 & 30,48 & 27,16 & 7,57 & 1,05 \\
\hline $\mathrm{HCP}_{05}$ & 0,38 & 0,94 & 0,57 & 0,83 & 0,70 & 0,11 & 0,51 & 0,23 \\
\hline \multicolumn{9}{|c|}{ Blé tendre : Scham-2 } \\
\hline $\begin{array}{l}\text { Témoin: sans } \\
\text { herbicides }\end{array}$ & 5,46 & 15,15 & 24,04 & 30,13 & 31,02 & 28,30 & 9,29 & 2,15 \\
\hline $\begin{array}{l}\text { 2,4-D+ Lontrel } \\
\text { en post-levée }\end{array}$ & 5,30 & 16,07 & 25,37 & 31,62 & 32,11 & 29,76 & 9,82 & 2,01 \\
\hline $\begin{array}{l}\text { Quartz super } \\
\text { en pré-levée }\end{array}$ & 5,49 & 16,58 & 25,71 & 32,25 & 33,08 & 30,11 & 10,29 & 1,93 \\
\hline $\begin{array}{l}\text { Quartz super } \\
\text { en post-levée }\end{array}$ & 5,29 & 16,04 & 25,39 & 31,73 & 32,35 & 29,74 & 9,90 & 2,05 \\
\hline $\mathrm{HCP}_{05}$ & 0,375 & 0,584 & 0,704 & 0,858 & 1,116 & 0,937 & 0,874 & 0,122 \\
\hline \multicolumn{9}{|c|}{ Blé dur : Bykhys-1 } \\
\hline $\begin{array}{l}\text { Témoin: sans } \\
\text { herbicides }\end{array}$ & 4,94 & 14,14 & 20,16 & 26,94 & 28,05 & 26,69 & 5,92 & 2,47 \\
\hline $\begin{array}{l}\text { 2,4-D+ Lontrel } \\
\text { en post-levée }\end{array}$ & 4,96 & 15,26 & 21,32 & 28,23 & 29,19 & 27,31 & 5,64 & 2,51 \\
\hline $\begin{array}{l}\text { Quartz super } \\
\text { en pré-levée }\end{array}$ & 5,28 & 15,88 & 22,33 & 29,04 & 29,99 & 27,95 & 5,19 & 2,3 \\
\hline $\begin{array}{l}\text { Quartz super } \\
\text { en post-levée }\end{array}$ & 4,93 & 15,23 & 21,77 & 28,20 & 29,20 & 27,33 & 5,63 & 2,52 \\
\hline $\mathrm{HCP}_{05}$ & 0,438 & 1,33 & 0,987 & 1,051 & 0,973 & 0,735 & 0,268 & 0,198 \\
\hline \multicolumn{9}{|c|}{ Blé dur : Homari } \\
\hline $\begin{array}{l}\text { Témoin: sans } \\
\text { herbicides }\end{array}$ & 4,19 & 12,1 & 16,82 & 24,37 & 25,64 & 23,84 & 4,57 & 1,94 \\
\hline $\begin{array}{l}\text { 2,4-D+ Lontrel } \\
\text { en pos-tlevée }\end{array}$ & 4,17 & 13,24 & 18,97 & 25,07 & 26,48 & 24,4 & 4,71 & 1,83 \\
\hline $\begin{array}{l}\text { Quartz super } \\
\text { en pré-levée }\end{array}$ & 4,57 & 14,15 & 19,79 & 25,88 & 27,34 & 25,03 & 4,33 & 1,6 \\
\hline $\begin{array}{l}\text { Quartz super } \\
\text { en post-levée }\end{array}$ & 4,24 & 13,31 & 19,12 & 25,18 & 26,44 & 24,49 & 4,84 & 1,94 \\
\hline $\mathrm{HCP}_{05}$ & 0,232 & 1,046 & 1,74 & 0,763 & 0,86 & 0,674 & 0,3 & 0,44 \\
\hline
\end{tabular}

Influence des herbicides sur les principaux paramètres physiologiques de la productivité des semis des blés tendres (Triticum aestivum L.) et durs (Triticum durum Desf.): Les principaux paramètres physiologiques de la productivité des semis étudiés ont été le potentiel photosynthétique, la biomasse, le rendement net de la photosynthèse et l'indice de récolte (tableau 4).Le potentiel photosynthétique ou rendement net de la photosynthèse est le rapport $\mathrm{du}$ rendement biologique sur l'accumulation journalière de matière sèche [Chatilov et al., 1987). La variété Lyba s'est démarquée par un potentiel photosynthétique peu élevé $\left(0,96\right.$ million $\left.\mathrm{m}^{2} \mathrm{ha} \mathrm{a}^{-1} / \mathrm{jour}\right)$. Les herbicides 

de la productivité et les composantes essentielles de rendement des blés tendres (Triticum aestivum $L$.) et durs (Triticum durum Desf.)

utilisés n'ont pas amélioré le potentiel photosynthétique. Apparemment, l'affectation de l'appareil foliaire à la phase (floraison-maturité laiteuse) par la rouille jaune du blé ( $P$. striiformis) a empêché l'amélioration du potentiel photosynthétique. La variété Scham-2 a enregistrée un potentiel photosynthétique de 1,02 million $\mathrm{m}^{2} /$ ha/jour soit une hausse de $6,3 \%$ qui n'a pas varié avec les herbicides. Les blés durs Bykhys-1et Homari ont enregistré sensiblement un même potentiel photosynthétique, soit respectivement 1,05 et 1,08 million $\mathrm{m}^{2}$ ha-1-jour. Les herbicides employés ont été sans effet. Cependant, chez les blés durs, la résistance à la rouille jaune du blé et une période végétative plus longue ont contribué à asseoir un potentiel photosynthétique plus élevé. Ainsi, le blé Bykhys-1 a été supérieur par son potentiel photosynthétique au blé Lyba de $9,3 \%$ et à la variété Scham-2 de 10,4\%, tandis que la variété Homari a connu des hausses de $12 \%$ et $13,5 \%$ sur ces deux variétés. Les biomasses enregistrées ont varié en fonction des variétés de blés avec cependant un impact positif des herbicides utilisés. Ainsi, dans les traitements témoins, il n'ya pas de différence notable dans les biomasses des blés tendres Lyba (6,42 tha$\left.{ }^{1}\right)$ et Scham-2 $\left(6,62 \mathrm{t} \mathrm{ha}^{-1}\right)$. Les herbicides utilisés ont permis des hausses de biomasses de $8,7 \%$ à $1,7 \%$ (Lyba) et de 3,3\% à 6,5\% (Scham-2). Les blés durs (Bykhys-1, Homari) enregistrent respectivement 6,23 et $5,16 \mathrm{tha}^{-1}$ sans les herbicides. Cependant, seule la variété Bykhys-1 avec en moyenne 6,74 $\mathrm{tha}^{-1}$ $(8,1 \%)$ de gain de biomasse dans les traitements herbicides soutien une comparaison avec les blés tendres. Quant au blé Homari, malgré une croissance de biomasse de 10,8\%, soit 5,72 $\mathrm{tha}^{-1}$, son taux de biomasse est demeuré le plus faible de l'essai. Ce blé a aussi enregistré en moyenne la plus faible surface foliaire de l'essai. Le rendement net de la photosynthèse caractérise l'activité productive de l'appareil photosynthétique. Les herbicides appliqués ont permis des hausses de ce paramètre de 8,6\%$10,1 \%$ (Lyba) et 7,2\%-27,8\% (Scham-2) et respectivement de $7,5 \%-7,7 \%$ et $8,5 \%-10 \%$ pour les blés durs Bykhys-1 et Homari. La variation du rendement photosynthétique net a été en fonction du régime thermique, hydrique, nutrition des semis et de la surface de l'appareil foliaire. L'indice de récolte (RI) constitue le rapport du rendement grain sur le rendement biologique (Donald, 1968). Le RI varie dans les traitements témoins entre un minimum de $46,3 \%$ pour Bykhys- 1 et un maximum de $51,1 \%$ pour Homari, alors que pour les blés tendres Lyba et Scham-2, il est respectivement de $43,3 \%$ et $50,2 \%$, soit de 1\%-7\% inférieur. Indépendamment des variétés, l'effet des herbicides a été nul sur les RI des blés Scham-2 et Homari et insignifiant sur celui de la variété Bykhys-1. Le blé Lyba est l'unique variété qui a enregistrée en moyenne un indice de récolte de $45 \%$ dans les traitements herbicides soit $4 \%$ de hausse par rapport au témoin. 
Tableau 4: Influence des herbicides sur les principaux paramètres physiologiques de la productivité des cultures de blés tendres et durs.

\begin{tabular}{|c|c|c|c|c|c|c|}
\hline Traitements & $\begin{array}{c}\text { Surface foliaire } \\
\text { maximale } \\
\text { (milliers } \mathrm{m}^{2} \mathrm{ha}^{-1} \text { ) }\end{array}$ & $\begin{array}{c}\text { Potentiel } \\
\text { Photosynthétique } \\
\text { des semis } \\
\text { (millions } \mathrm{m}^{2} \mathrm{ha}^{-1} / \text { jour) }\end{array}$ & $\begin{array}{l}\text { Rendement } \\
\text { biologique } \\
\text { (t ha-1) }\end{array}$ & $\begin{array}{l}\text { Accumulation journalière } \\
\text { de matière sèche } \\
\text { (g ha-1/ jour) }\end{array}$ & $\begin{array}{l}\text { Rendement } \\
\text { Grains } \\
\text { (t ha-1) }\end{array}$ & $\begin{array}{l}\text { Indice de } \\
\text { récolte } \\
\text { (RI); \% }\end{array}$ \\
\hline \multicolumn{7}{|c|}{ Blé tendre: Lyba } \\
\hline Témoin: sans herbicides & 29,48 & 0,96 & 6,42 & 6,69 & 2,78 & 43,30 \\
\hline 2,4-D+ Lontrel en post-levée & 30,43 & 0,96 & 7,06 & 7,35 & 3,12 & 44,20 \\
\hline Quartz super en pré-levée & 30,35 & 0,96 & 6,98 & 7,27 & 3,21 & 46,0 \\
\hline Quartz super en post-levée & 30,48 & 0,97 & 7,15 & 7,37 & 3,20 & 44,80 \\
\hline $\mathrm{HCP}_{05}$ & 0,70 & 0,05 & 0,38 & 0,16 & 0,24 & 2,00 \\
\hline \multicolumn{7}{|c|}{ Blé tendre: Scham-2 } \\
\hline Témoin: sans herbicides & 31,02 & 1,02 & 6,62 & 5,28 & 3,21 & 50,20 \\
\hline 2,4-D+ Lontrel en post-levée & 32,11 & 1,02 & 7,07 & 6,34 & 3,39 & 50,10 \\
\hline Quartz super en pré-levée & 33,08 & 1,02 & 7,05 & 5,66 & 3,43 & 50,50 \\
\hline Quartz super en post-levée & 32,35 & 1,01 & 7,10 & 6,75 & 3,42 & 50,20 \\
\hline $\mathrm{HCP}_{05}$ & 1,04 & 0,09 & 0,27 & 0,13 & 0,12 & 0,73 \\
\hline \multicolumn{7}{|c|}{ Blé dur: Bykhys-1 } \\
\hline Témoin: sans herbicides & 28,05 & 1,05 & 6,23 & 5,93 & 2,87 & 46,30 \\
\hline 2,4-D+ Lontrel en post-levée & 29,19 & 1,05 & 6,71 & 6,39 & 3,13 & 46,80 \\
\hline Quartz super en pré-levée & 29,99 & 1,06 & 6,76 & 6,38 & 3,19 & 47,30 \\
\hline Quartz super en post-levée & 29,20 & 1,06 & 6,76 & 6,38 & 3,16 & 46,90 \\
\hline $\mathrm{HCP}_{05}$ & 0,97 & - & 0,31 & - & 0,16 & 0,72 \\
\hline \multicolumn{7}{|c|}{ Blé dur: Homari } \\
\hline Témoin: sans herbicides & 25,64 & 1,08 & 5,16 & 4,78 & 2,64 & 51,10 \\
\hline 2,4-D+ Lontrel en post-levée & 26,48 & 1,09 & 5,66 & 5,19 & 2,90 & 51,10 \\
\hline Quartz super en pré-levée & 27,34 & 1,09 & 5,72 & 5,25 & 2,93 & 51,30 \\
\hline Quartz super en post-levée & 26,44 & 1,10 & 5,78 & 5,26 & 2,95 & 50,90 \\
\hline $\mathrm{HCP}_{05}$ & 0,86 & - & 0,35 & -- & 0,17 & 0,35 \\
\hline
\end{tabular}




\section{DISCUSSION}

Influence des herbicides sur les principaux paramètres physiologiques de la productivité des blés tendres (Triticum aestivum L.) et durs (Triticum durum Desf.): Indépendamment des herbicides appliqués, les blés tendres Lyba et Scham-2 ont enregistré les plus fortes surfaces foliaires, respectivement 30,42 milles $\mathrm{m}^{2} \mathrm{ha}^{-1}$ et 32,51 milles $\mathrm{m}^{2} \mathrm{ha}^{-1}$ en moyenne. L'analyse de l'effet des herbicides indique une réaction plus sensible des blés Scham-2 et Bykhys-1 qui ont enregistré les plus fortes hausses de surface foliaire, soit un taux de $4,8 \%$ et $5,1 \%$ respectivement. De par sa particularité biologique, le blé local Lyba, très sensible à la rouille jaune a subit une forte infestation au stade laiteuxcireux de sa surface foliaire pendant l'année humide et froide (1998) de l'étude entrainant une chute de 10-15 milles $\mathrm{m}^{2} \mathrm{ha}^{-1}$ de l'appareil foliaire. L'effet des herbicides a été insignifiant pour cette variété. Au contraire, les variétés Scham-2, Bykhys-1 et Homari ont montré une résistance forte aux espèces de rouille brune et jaune. Pour certaines catégories de cultures, des études menées par De Jesus et al. (2001) et Robert et al. (2005) ont établi une relation entre la croissance, ou le rendement et le rayonnement intercepté par l'appareil foliaire. Selon Chatilov et al. (1987), le blé qui assimile environ $0,9 \%$ des radiations reçues devrait en retour enregistrer des surfaces foliaires maximales de 4-5 fois supérieure à la surface occupée par les semis soit, $40-50$ milles $\mathrm{m}^{2} \mathrm{ha}^{-1}$ dans les conditions hydriques favorables pour assurer des rendements stables. Dans la pratique, l'ensemble des zones de production des blés sont situées dans des régions insuffisamment arrosées et les semis des blés enregistrent des surfaces foliaires très souvent inférieur à 40 milles $\mathrm{m}^{2}$ ha-1. La connaissance de la dimension des principaux paramètres physiologiques de la productivité que sont le potentiel photosynthétique, la biomasse, le rendement net de la photosynthèse et l'indice de récolte (RI) est essentielle dans la mesure où ils sont déterminant dans le rendement de la culture. Le développement et la croissance de l'appareil foliaire dépendent de la dimension du potentiel photosynthétique du semis (millions de $\mathrm{m}^{2}$ ha-1/jour). Celui-ci reflète la somme de la surface foliaire des semis par unité de surface au cours de la végétation et reste lié aux rendements biologique et utile (Jeuffroy et al., 2000). Le potentiel photosynthétique le plus faible a été enregistré par le blé tendre Lyba indépendamment des herbicides employés (0.96 millions $\mathrm{m}^{2}$ ha-1-jour). La sensibilité de cette variété à la rouille brune et à la septoriose
(Mycosphaerella graminicola) pourrait être la principale cause de cette chute. La rouille brune provoquerait une diminution des surfaces foliaires vertes entrainant une baisse de la photosynthèse des tissus infectés tandis que la septoriose induirait une sénescence accélérée des feuilles (El Jarroudi, 2005; Robert et al., 2006). II y a une forte relation de dépendance entre le potentiel photosynthétique et les rendements biologique et grain. Cependant, cette corrélation est très souvent influencée par l'action des conditions climatiques sur la productivité de l'appareil photosynthétique (Demotes-Mainard and Jeuffroy, 2001). La valeur de la matière sèche journalière accumulée par les semis tout comme la taille de l'appareil foliaire est déterminant dans la valeur de la récolte (Nichiporovich, 1961; Chatilov et al., 1987). Indépendamment des traitements herbicides, les blés tendres Lyba $(8,6 \%-10,1 \%)$ et Scham-2 (7,2\%-27,8\%) ont enregistré la plus forte accumulation journalière de matière sèche. La valeur journalière de matière sèche est le résultat de l'activité effective de la surface foliaire pendant la période végétative. Elle serait fonction des régimes de température, d'eau, de nutrition, et surtout de la dimension de la surface foliaire (Bancal et al., 2007). D'autre part, il est observé chez ces blés tendres indépendamment des traitements les plus forts taux accumulés de matière sèche, soit des hausses de 8,7\%-11,7\% (Lyba) et de 6,5\%-3,3\% (Scham-2). Quant au blé dur Homari, malgré une croissance de biomasse de 10,8\%, son taux de biomasse est demeuré le plus faible de l'essai. Ce blé a aussi enregistré en moyenne la plus faible surface foliaire de l'essai. Le nombre de talles/pied de 3 à 4 pour ce blé est réduit à 1,7-2,2 talles/pied au cours de l'année sèche et chaude(1999); ceci a entrainé une chute de la surface foliaire formée et de la matière sèche accumulée par ce blé. Visiblement ces particularités biologiques (nombre faible de talles/pied) et physiologiques (mauvaise adaptation aux conditions climatiques) ont contribué à réduire la matière sèche de ce blé. Le rendement biologique, bien que lié à la dimension du potentiel photosynthétique, n'est cependant pas entièrement proportionnel; cela s'explique par la variation de la productivité de l'appareil photosynthétique en fonction des changements des conditions du milieu (Triboi and Triboi-Blondel, 2002). Une augmentation de la masse sèche végétale globale par unité de surface serait un préalable pour accroitre la production (Shearman et al., 2005). Ceci peut s'obtenir soit par une augmentation de la durée de l'activité 
photosynthétique après la floraison (Borrell et al., 2000; Spano et al., 2003) ou bien par la prolongation de la durée de vie de la surface verte de la dernière feuille (Dimmock and Gooding, 2002; Pepler et al., 2005). Les indices de récolte (RI) observés chez les variétés tendres Lyba et Scham-2 ont été plus faibles, indépendamment des traitements herbicides. Ce résultat en accord avec les travaux de Donald (1968), Hamidou et al. (2005) et Bahloudi et al. (2008) et serait probablement dû à un détournement des produits de la photosynthèse vers l'appareil végétatif (tige + racine) au détriment du remplissage des grains. La supériorité des blés durs Bykhys-1 et Homari à accumuler les assimilâts est la conséquence d'une plus grande stabilité dans les rendements et a une supériorité des indices de récolte respectifs. En outre, de nombreux travaux effectués au cours des dix dernières années ont montré un lien significatif entre l'accroissement de RI et l'augmentation du nombre de grains par mettre carré, alors que la masse sèche du grain de blé est restée stable ou a même légèrement diminué (Donmez et al., 2001; Trottet and Doussinauld, 2002).

Influence des herbicides sur les composantes essentielles de rendement des blés tendres (Triticum aestivum L.) et durs (Triticum. Durum Desf.): L'impact positif des herbicides utilisés sur les paramètres physiologiques de la productivité et dans une moindre mesure associés aux particularités biologiques de chaque variété de blé ont eu un effet positif sur la structure des composantes du rendement. Les présents résultats ont montré une meilleure réaction des blés tendre (Lyba) et dur (Homari) aux herbicides appliqués. Selon Fontaine et al. (2009) et Lecomte et al. (2008), ces variétés à faible tallage et à courte tige seraient moins concurrentielles aux adventices comparées aux variétés à fort tallage (Scham-2) et à haute tige (Bykhys-1). En général, les herbicides utilisés ont permis chez toutes les variétés la conservation d'une meilleure densité de peuplement à la récolte, ce qui pourrait accroitre le rendement grain. De même, le rendement grain des différents blés a été plus dépendant du nombre de grains formés par plant plus que le poids individuel d'un grain. Ces résultats corroborent avec ceux obtenus par nombres d'auteurs qui ont démontré que le rendement était souvent très bien corrélé au nombre de grains formés alors qu'il ne l'est pas avec le poids individuel grain (Sinclair and Jamieson, 2006). Cependant, les relations observées entre nombre de grains et le rendement sont empiriques car le nombre de grains n'est pas le facteur déterminant de leur masse sèche (Slama et al., 2005). 
Garané et al. J. Appl. Biosci. Influence des herbicides sur les principaux paramètres physiologiques de la productivité et les composantes essentielles de rendement des blés tendres (Triticum aestivum L.) et durs (Triticum durum Desf.)

Tableau 5: Influence des herbicides sur les composantes de la structure de rendement des variétés de blés tendres et durs.

\begin{tabular}{|c|c|c|c|c|c|c|c|c|c|}
\hline \multirow[b]{2}{*}{ Traitements } & \multirow{2}{*}{$\begin{array}{l}\text { Hauteur } \\
\text { des } \\
\text { plants } \\
\text { (cm) }\end{array}$} & \multicolumn{2}{|c|}{ Nombre par plant de } & \multirow[b]{2}{*}{$\begin{array}{c}\text { Nombre de } \\
\text { grains par } \\
\text { plant }\end{array}$} & \multirow[b]{2}{*}{$\begin{array}{l}\text { Poids de } \\
\text { grains par } \\
\text { plant (g) }\end{array}$} & \multirow{2}{*}{$\begin{array}{c}\text { Poids de } \\
1000 \\
\text { grains } \\
\text { (g) }\end{array}$} & \multicolumn{2}{|c|}{$\begin{array}{l}\text { Nombre par mettre } \\
\text { carré de }\left(\mathrm{m}^{2}\right)\end{array}$} & \multirow{2}{*}{$\begin{array}{l}\text { Poids de } \\
\text { grains par } \\
\text { mettre } \\
\text { carré } \\
\left(\mathrm{g} / \mathrm{m}^{2}\right)\end{array}$} \\
\hline & & Talles & $\begin{array}{c}\text { Talles -utiles } \\
\text { (peuplement- } \\
\text { épis) }\end{array}$ & & & & Plants & $\begin{array}{l}\text { Talles - } \\
\text { utiles }\end{array}$ & \\
\hline \multicolumn{10}{|c|}{ Blé tendre: Lyba } \\
\hline Témoin: sans herbicides & 78,00 & 1,17 & 1,03 & 23,20 & 0,79 & 34,10 & 343,0 & 353,0 & 271,0 \\
\hline $2,4-D+$ Lontrel en post-levée & 80,10 & 1,20 & 1,07 & 24,70 & 0,87 & 35,20 & 352,0 & 377,0 & 306,3 \\
\hline Quartz super en pré-levée & 81,20 & 1,20 & 1,05 & 25,40 & 0,89 & 35,10 & 354,0 & 372,0 & 315,1 \\
\hline $\begin{array}{l}\text { Quartz super en post-levée } \\
\mathrm{HCP}_{05}\end{array}$ & $\begin{array}{c}80,40 \\
1,68\end{array}$ & $\begin{array}{l}1,20 \\
0,02\end{array}$ & $\begin{array}{l}1,06 \\
0,02 \\
\end{array}$ & $\begin{array}{c}25,20 \\
1,27\end{array}$ & $\begin{array}{l}0,89 \\
0,05 \\
\end{array}$ & $\begin{array}{c}35,30 \\
0,83\end{array}$ & $\begin{array}{c}353,0 \\
8,20\end{array}$ & $\begin{array}{l}374,0 \\
14,11\end{array}$ & $\begin{array}{l}314,2 \\
12,17\end{array}$ \\
\hline \multicolumn{10}{|c|}{ Blé tendre: Scham-2 } \\
\hline Témoin: sans herbicides & 76,30 & 2,28 & 1,76 & 41,90 & 1,50 & 35,80 & 350,0 & 616,0 & 525,0 \\
\hline 2,4-D + Lontrel en post-levée & 75,30 & 2,35 & 1,77 & 44,00 & 1,60 & 36,40 & 348,0 & 616,0 & 557,0 \\
\hline Quartz super en pré-levée & 73,30 & 2,40 & 1,78 & 43,70 & 1,63 & 37,30 & 352,0 & 627,0 & 574,8 \\
\hline Quartz super en post-levée & 76,30 & 2,38 & 1,76 & 44,30 & 1,61 & 36,30 & 350,0 & 616,0 & 563,5 \\
\hline $\mathrm{HCP}_{05}$ & 1,55 & 0,10 & 0,03 & 1,25 & 0,36 & 0,36 & 1,19 & 7,84 & 10,36 \\
\hline \multicolumn{10}{|c|}{ Blé dur: Bykhys-1 } \\
\hline Témoin: sans herbicides & 67,00 & 2,23 & 1,40 & 34,80 & 1,37 & 39,40 & 350,0 & 490,0 & 479,5 \\
\hline 2,4-D + Lontrel en post-levée & 67,20 & 2,32 & 1,43 & 36,70 & 1,50 & 40,90 & 350,0 & 501,0 & 525,0 \\
\hline Quartz super en pré-levée & 65,60 & 2,17 & 1,46 & 36,80 & 1,49 & 40,50 & 357,0 & 521,0 & 532,0 \\
\hline Quartz super en post-levée & 67,70 & 2,23 & 1,40 & 37,60 & 1,52 & 40,40 & 349,0 & 489,0 & 530,8 \\
\hline $\mathrm{HCP}_{05}$ & 1,35 & 0,10 & 0,29 & 1,44 & 0,08 & 0,66 & 2,63 & 7,19 & 6,35 \\
\hline \multicolumn{10}{|c|}{ Blé dur: Homari } \\
\hline Témoin: sans herbicides & 57,30 & 2,43 & 1,56 & 34,1 & 1,26 & 37,00 & 350,0 & 546,0 & 441,0 \\
\hline 2,4-D + Lontrel en post-levée & 58,00 & 2,43 & 1,56 & 36,8 & 1,39 & 37,80 & 350,0 & 546,0 & 486,5 \\
\hline Quartz super en pré-levée & 56,70 & 2,40 & 1,53 & 36,7 & 1,39 & 38,00 & 354,0 & 542,0 & 492,1 \\
\hline Quartz super en post-levée & 58,70 & 2,46 & 1,60 & 37,5 & 1,41 & 37,80 & 350,0 & 563,0 & 493,5 \\
\hline $\mathrm{HCP}_{05}$ & 1,19 & 0,10 & 0,07 & 1,79 & 0,08 & 0,60 & 1,19 & 14,92 & 6,91 \\
\hline
\end{tabular}



(Triticum durum Desf.)

\section{CONCLUSION}

L'efficacité des herbicides utilisés est appréciée à partir de l'amélioration des paramètres physiologiques de la productivité et par la hausse des composantes de la structure du rendement des blés étudiés. Les résultats ont montré chez les blés tendres une meilleure réaction aux herbicides utilisés de la variété locale Lyba à faible tallage. Ce blé a enregistré une hausse plus sensible de toutes ses composantes de rendement. $\mathrm{Au}$ contraire, les herbicides ont été moins efficaces dans les parcelles du blé Scham-2 à tallage fort; ses composantes du rendement ont connu une

\section{REFERENCES BIBLIOGRAPHIQUES}

Assem N., El Hafid L., Haloui B. and El Atmani. 2006. Effets du stress hydrique appliqué au stade trois feuilles sur le rendement en grains de dix variétés de blé cultivées au Maroc oriental. Science et changements planétaires. Sécheresse, 17(4): 499-505.

Asseng S., Turner N. C., Ray J. D., and Keating B. A. 2002. A simulation analysis that redicts the influence of physiological traits on the potential yield of wheat. European Journal of Agronomy, 17: 123-141.

Bahloudi F., Bouzerzour $H$. and Benmahammed A. 2008. Effets de la vitesse et de la durée du remplissage du grain ainsi que de l'accumulation des assimulats de la tige dans l'élaboration du rendement du blé dur (Tr. durum Desf.) dans les conditions de culture des hautes plaines orientales d'Algérie. Biotechnology Agronomy Society and Environment, 12: 31-39.

Bahlouli. F., Bouzerzour H., Benmahammed A. and Hassous K. L. 2005. Selection of high yielding and risk efficient durum wheat (Tr. durum Desf.) cultivars under semi arid conditions. J. Agro.; 4: 360-365.

Bancal M. O., Robert C. and Ney B. 2007. Modelling wheat growth and yield losses from late epidemics of foliar diseases using loss of green leaf area per layer and pre-anthesis reserves. Annals of Botany, 4: 777-789.

Bàrberi $P$ Weed management in organic agriculture: are we addressing the right issues. Weed Research, 2002; 42: 177-193.

Borrell A. K., Hammer G. L. and Henzell R. G. 2000. Does maintaining green leaf area in sorghum amélioration moins sensible. Pour les blés durs, la variété Homari à tige courte a été plus réceptive aux désherbants appliqués. Indépendamment du type de blé, les variétés à faible tallage et à tige courte qui sont moins concurrentielles aux adventices ont montré une plus meilleure réaction aux herbicides utilisée. Les herbicides employés ont permis en général pour toutes les variétés la conservation d'une meilleure densité de peuplement à la récolte, ce qui pourrait accroitre le rendement grain.

improve yield under drought? II. Dry matter production and yield. Crop Science, 40: 10371048.

Chatilov I. S., Charov A. F., and Ratysova L. A., 1987. Activité photosynthétique et productivité du blé d'hiver dans la région central de la zone «nichernozièm» de la Russie. Publ. Acad. Agricole Tymirazeef, 1: 13-15.

Cummins J. 2009. Glyphosate Resistance in weeds. Institute of Science in Society. European Journal of Agronomy, 31(1): 62-72.

Darui A., Boukroute A., Kajeiou A. and Berrichi A. 2011. Effet de l'irrigation d'appoint sur le rendement d'une culture de blé tendre (Triticum aestivum L.) (variété Rajae) au Maroc Oriental. Nature et Technologie. 5: 8086.

De Jesus W.C., Ribeiro Jr., do Vale X., Coelho R. R. and Costa L. C. 2001. Comparison of two methods for estimating leaf area index on common Bean. Agronomy Journal.; 93: 989991.

Demotes-Mainard S. and Jeuffroy M. H. 2001. Incorporating radiation and nitrogen nutrition into a model of kernel number in wheat. Crop Science 41(2): 415-423.

Dimmock J. P. R. E. and Gooding M. J. 2002. The effects of fungicides on rate and duration of grain filling in winter wheat in relation to maintenance of flag leaf green area. Journal of Agricultural Science, 138: 1-16.

Donald C. M. 1968. The breeding of crop ideotypes. Euphytica, 17: 385-403.

Donmez E., Sears R. G., Shroyer J. P. and Paulsen G. M. 2001. Genetic gain in yield attributes of 
Garané et al. J. Appl. Biosci. Influence des herbicides sur les principaux paramètres physiologiques de la productivité et les composantes essentielles de rendement des blés tendres (Triticum aestivum L.) et durs (Triticum durum Desf.)

winter wheat in the great plains. Crop Science, $41: 1412$ - 1419.

Doré T., Le Bail M., Martin P., Ney B. and RogerEstrade J. 2006. Conclusion générale, in: "L'agronomie aujourd'hui", Doré T., Le Bail M., Martin P., Ney B., Roger-Estrade J. (eds), Editions Quae. 311-313.

El Jarroudi M. 2005. Évaluation des paramètres épidémiologiques des principales maladies cryptogamiques affectant les feuilles du blé d'hiver au Grand-Duché de Luxembourg: Calibration et validation d'un modèle de prévision. Thèse de doctorat Université de Liège, Département des Sciences et Gestion de l'Environnement.

Farineau J. and Morot-Gaudry J. F. 2006. Feuilles et chloroplastes. In La photosynthèse, Processus physiques, moléculaires et physiologiques. Synthèses, 1: 93-99.

Fontaine L., Bernicot M., Rolland B. and Poiret L. 2009. Des variétés rustiques concurrentes des adventices pour l'agriculture durable, en particulier l'agriculture biologique. Innovations agronomiques, 115-124.

Gooding M. J., Dimmock J. P. R. E., France J. and Jones S. A. 2000. Green leaf area decline of wheat flag leaves: the influence of fungicides and relationships with mean grain weight and grain yield. Annals of Applied Biology, 136: 7784.

Hamidou F., Dicko M. H., Traoré A. S. and Ginko S. 2005. Adaptative response of two varieties cowpeas to water stress. Cahiers d'études et de recherches francophone, 14: 161-167.

Jeuffroy M.H., Barré C., Bouchard C., DesmotesMainard S., Devienne-Barret F. L., Girard M. and Recous S. 2000. Fonctionnement d'un peuplement de blé en condition de nutrition azotée sub-optimale. In: Fonctionnement des peuplements végétaux sous contraintes environnementales. INRA, Paris.

Kurstjens D. A. G. 2007. Precise tillage Systems for enhanced non-chemical weed management. Soil and Tillage. Research, 97: 293-305.

Lecomte C., Heumez E. and Pluchard P. 2008. Identification de différences génotypiques dans la réponse aux contraintes environnementales: cas de la concurrence due aux mauvaises herbes dans une culture de blé tendre d'hiver. In: Maillard P., Bonhomme R.
(Eds.), Fonctionnement des peuplements végétaux sous contraintes environnementales. INRA, Paris, 2000; 539-558, Maîtrise de la flore et éléments de l'itinéraire technique. Innovations Agronomiques, 3: 27-41.

Nichiporovich A. A. (1961). De la formation à la productivité de l'appareil photosynthétique de différentes cultures au cours de la période végétative. Physiologie des plantes, 8(1): 4753.

Pepler S., Gooding M. J., Ford K. E. and Ellis R. H. 2005. A temporal limit to the association between flag leaf life extension by fungicides and wheat yields. European Journal of Agronomy, 22: 363-373.

Prieur L. 2010. Maîtriser les adventices dans les cultures biologiques. Agronomie, Fiche N ${ }^{\circ} 1, p$. 4.

Ramdani A. 2004. Impact of Spanish and Italian breeding activities on durum wheat yield and associated morpho-physiological and quality traits throughout the 20th century. Thèse de Ph.D. Département de Production végétale, Université de Leida, Esp.

Robert C., Bancal M. O., Lannou C. and Ney B. 2006. Quantification of the effects of Septoriatritici blotch on wheat leaf gas exchange with respect to lesion age, leaf number, and leaf nitrogen status. Journal of Experimental Botany. 57: 225-234.

Robert C., Bancal M. O., Ney B. and Lannou C. 2005. Wheat leaf photosynthesis loss due to leaf rust, with respect to lesion development and leaf nitrogen status. New Physiologist, 165: 227-241.

Shearman V. J., Sylvester-Bradley R., Scott R. K. and Foulkes M. J. 2005. Physiological processes associated with wheat yield progress in the UK. Crop Science, 45: 175-185.

Sinclair T.R. and Jamieson P. D. 2006. Grain number, wheat yield, and bottling beer: An analysis, Field. Crops Research., 98: 60-67.

Slama A., Ben Salem M., Ben Naceur M. and Zid B. 2005. Les céréales en Tunisie: production, effet de la sécheresse et mécanismes de résistance. Sécheresse, 16(3): 225-229.

Spano G., Fonzo N. D., Perrotta C., Platani C., Ronga G., Lawlor D. W., Napier J. A. and Shewry P. R. 2003. Physiological characterization of 'stay 

la productivité et les composantes essentielles de rendement des blés tendres (Triticum aestivum L.) et durs (Triticum durum Desf.)

green' mutants in durum wheat. Journal of Experimental Botany, 54:1415-1420.

Triboi E. and Triboi-Blondel A. M. 2002. Productivity and grain or seed composition: a new approach to an old problem - invited paper. European Journal of Agronomy, 16: 163-186.

Trottet M. and Doussinault G. 2002. Analyse du progrès génétique chez le blé tendre au cours du XXème siècle. Le Sélectionneur Français, 53: $3-13$.

Turner R. J., Davies G., Moore H., Grundy A. C. and Mead A. 2006; Organic weed management: A review of the current UK farmer perspective. Coventry: Crop protection, 26(3): 377-382.

Valantin-Morisson M., Guichard L. and Jeuffroy M. H. 2008. Comment maîtriser la flore adventice des grandes cultures à travers des éléments de l'itinéraire technique. Innovations agronomiques. 3: 27- 41 